\title{
Limits on O VI Emission from the Shocked Circumstellar Gas of SN 1987A
}

\author{
George Sonneborn*, Rosina C. Iping*, ${ }^{* \dagger}$, Peter Lundqvist** and Claes \\ Fransson** \\ *Laboratory for Observational Cosmology, NASA/Goddard Space Flight Center, Code 665 , \\ Greenbelt, MD, USA \\ Department of Physics, Catholic University of America, Washington, DC, USA \\ ${ }^{*}$ Stockholm Observatory, Stockholm, Sweden
}

\begin{abstract}
.
The Far Ultraviolet Spectroscopic Explorer (FUSE) was used to search for emission from the shock interaction of the ejecta of SN 1987A with its circumstellar material. FUSE observations of SN 1987A between 2000 and 2007 did not detect broad O VI emission. However, O VI emission was detected in 2000-2001 with a narrow line width $\left(F W H M<35 \mathrm{~km} \mathrm{~s}^{-1}\right)$ and a heliocentric radial velocity of $+280 \mathrm{~km} \mathrm{~s}^{-1}$. This places the emitting gas at rest relative to the supernova and is interpreted as emission from unshocked circumstellar gas. This narrow emission had disappeared in 2007 (and possibly earlier) as a result of the advancing shock overtaking the $\mathrm{H}$ II region that was flash ionized by the supernova explosion in 1987.
\end{abstract}

Keywords: Supernovae, SN 1987A

PACS: $95.55 . \mathrm{Fw}, 95.85 . \mathrm{Mt}, 97.60 . \mathrm{Bw}, 98.38 . \mathrm{Mz}$

The spectroscopic signature of the collision of SN 1987A ejecta with its circumstellar material, UV resonance-line emission (e.g. N V, CIV, Si IV, with $F W H M \sim 300$ $\mathrm{km} \mathrm{s}^{-1}$ ), was detected in HST/STIS spectra (Sonneborn et al. 1998, Pun et al. 2002). Broad emission from optical coronal lines (e.g. [Fe X], [Fe XI], and [Fe XIV]) has also emerged since 2000 and increased exponentially in strength (Gröningsson et al. 2006). These results are generally consistent with shock-interaction models. O VI 1032-1038 A emission is expected to track the coronal lines and to be the primary cooling transition for the million-degree shocked gas. An accurate measurement of the OVI line strength would significantly improve current models of the shock interaction. The far-ultraviolet O VI 1032, $1038 \AA$ resonance lines are expected to be one of the key signatures of highly ionized gas in the circumstellar environment of SN 1987A.

SN 1987A was observed by the Far Ultraviolet Spectroscopic Explorer (FUSE) satellite in 2000, 2001 and 2007 with the HIRS slit (1.25×20 arcsec) and in 2002 and 2004 with the LWRS slit $(30 \times 30$ arcsec). The 2000 and 2001 spectra showed a very narrow O VI 1032 emission line profile ( $\left.F W H M<35 \mathrm{~km} \mathrm{~s}^{-1}\right)$ at a heliocentric radial velocity of $+280 \mathrm{~km} \mathrm{~s}^{-1}$ (see Fig. 1), indicating that the emitting gas is at rest relative to the SN. However, the narrow line width shows that is does not originate in the shock interaction zone.

The 2000 and 2001 FUSE observations used the HIRS aperture $(1.25 \times 20$ arcsec) in order to minimize the spectral contamination from the two B stars a few arc seconds from SN 1987A. Spectra obtained simultaneously in the other FUSE apertures, positioned 


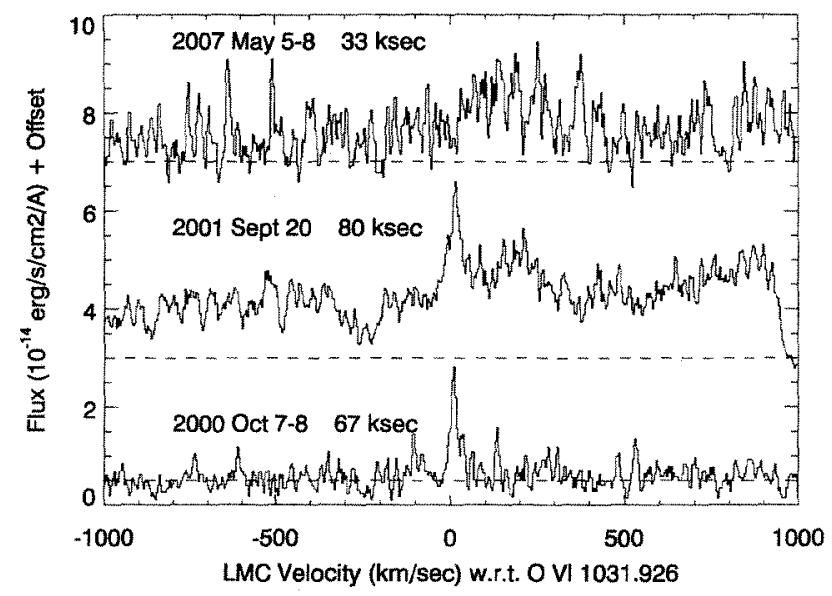

FIGURE 1. The OVI 1032 spectral region in three FUSE observations. The velocity zero point is $v_{\text {helio }}=+280 \mathrm{~km} \mathrm{~s}^{-1}$. All spectra have been processed with CalFUSE3. Narrow OVI is present in the HIRS spectrum (Oct. 2000).

about 100 arcsec from the HIRS slit, show nothing at the OVI wavelengths, eliminating diffuse emission in the LMC as a possible source of the narrow O VI feature. The narrow OVI emission probably originates from a region of lower density $\left(n_{e}<100 \mathrm{~cm}^{-3}\right)$, unshocked gas that was ionized by the supernova outburst in 1987. The flux in the narrow O VI feature is in agreement with recombination models (Lundqvist 1999) for the circumstellar material.

The last FUSE observation of SN 1987A was made on May 2007 (also with the HIRS aperture), shortly before the end of the mission. There is no convincing detection of broad O VI emission. This spectrum gives an upper limit on broad O VI emission from SN 1987A in 2007 of $\sim 8 \times 10^{-15} \mathrm{erg} \mathrm{cm}^{-2} \mathrm{~s}^{-1}$ in observed flux, or $\sim 1 \times 10^{-13} \mathrm{erg}$ $\mathrm{cm}^{-2} \mathrm{~s}^{-1}$ corrected for foreground Galactic and LMC extinction.

The 2007 spectrum also lacks the narrow O VI feature observed earlier. Its disappearance in a matter of several years is not likely to be the result of recombination 20 years after the initial ionization of the gas. Rather, the sudden decrease in the O VI emission is likely to be caused by the advance of the SN blast wave sweeping up the H II region interior to the inner circumstellar ring (see discussion of the circumstellar geometry in Lundqvist 1999).

Gröningsson et al. (2006) have modeled the shock interaction taking place in the inner ring and find good agreement with their observations of optical coronal lines of [Fe X], [Fe XI], and [Fe XIV] (see Figure 2). The cooling of the shocked gas occurs in a very thin region where the temperature drops from $\sim 10^{6} \mathrm{~K}$ to $\sim 10^{4} \mathrm{~K}$. O VI has the highest cooling rate, hence is a sensitive diagnostic of the cooling region. The $[\mathrm{Fe}$ 

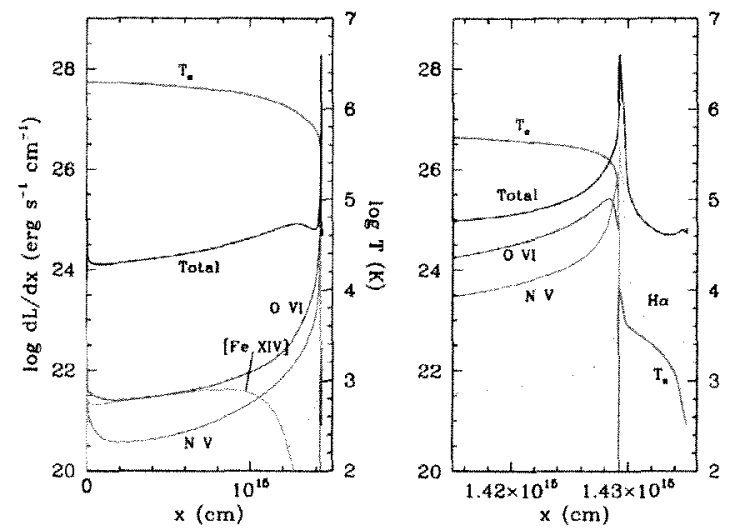

FIGURE 2. The cooling rate per unit distance for O VI, NV, $\mathrm{H} \alpha$, and [Fe XIV] (left-hand axis) and electron temperature (right-hand axis) are shown as a function of position behind a radiative shock with velocity $350 \mathrm{~km} \mathrm{~s}^{-1}$ and pre-shock density $10^{4} \mathrm{~cm}^{-3}$. The left-hand panel shows the total shock, while the right-hand panel shows the cooling region in more detail.

XIV] line mainly probes the immediate post-shock region and the $\mathrm{H} \alpha$ line the cool, photoionized region in front of the shock. OVI is predicted to track the exponential growth of the coronal lines and soft X-rays. The [Fe XIV] light curve (Gröningsson et al. 2006) indicated that O VI should increase significantly by the time of the 2007 May observation. However, no O VI emission from shocked gas was detected in the FUSE observations.

The fast radiative shock models of Allen et al. (2008) provide an additional check these calculations. For shocks with velocity 200 and $500 \mathrm{~km} \mathrm{~s}^{-1}$, N V should be present mainly for shocks closer to $200 \mathrm{~km} \mathrm{~s}^{-1}$. At $500 \mathrm{~km} \mathrm{~s}^{-1}$ the $\mathrm{N} \mathrm{V}$ relative abundance is low. The situation is very similar for O VI. For the narrow-line precursor, it is harder to produce $\mathrm{O}$ VI than $\mathrm{N} \mathrm{V}$, but the precursor is certainly dominated by $\mathrm{N}$ II and $\mathrm{O}$ III. The results of Allen et al. agree fairly well with the models of Göningsson et al. (2006), i.e., a sign of an advancing shock precursor is mainly provided by increasing narrow [O III] emission, or at least that [O III] emission falls off more slowly than other narrow lines. This is presented in more detail by Gröningsson et al. (2008).

\section{REFERENCES}

M. Allen, et al. arXiv:0805.0204 (2008).

P. Gröningsson, et al. Astron. \& Astrophys. 456, 581 (2006).

P. Gröningsson, et al. arXiv:0810:2661 (2008).

P. Lundqvist Astrophys. J. 511, 389 (1999).

J. Pun, et al. Astrophys. J. 572, 906 (2002).

G. Sonneborn et al. Astrophys. J. 492, L139 (1998). 